\title{
Three Poliovirus Sequences in the Human Genome Associated With Colorectal Cancer
}

\author{
STEVEN LEHRER ${ }^{1}$ and PETER H. RHEINSTEIN ${ }^{2}$ \\ ${ }^{1}$ Radiation Oncology, Mount Sinai Medical Center, New York, NY, U.S.A.; \\ ${ }^{2}$ Severn Health Solutions, Severna Park, MD, U.S.A.
}

\begin{abstract}
Background/Aim: In a previous study we analyzed poliomyelitis incidence in US states, before the introduction of the polio vaccine. We noted an inverse correlation between polio incidence in US states, between 1937 and 1951, and colorectal cancer incidence (2005-2009). To further elucidate the role that poliovirus could play in colorectal cancer, the full human genome for poliovirus sequences was analyzed using the UCSC Genome Browser. Materials and Methods: BLAT, the Blast-Like Alignment Tool of the UCSC Genome Browser, was used to compare the poliovirus genome to the human genome. Results: BLAT revealed three poliovirus sequences in three chromosomes: Chromosome 20p12.1 (MACROD2 gene), chromosome 1p13.3 (SLC25A24 gene), and chromosome 2p25.1. Conclusion: Poliovirus sequences in the human genome may contribute to programmed cell death and lysis of colorectal cancer cells. A recombinant poliovirus, incapable of reverting to neurovirulence, might be given orally at intervals as a colorectal cancer vaccine to prevent colorectal cancer.
\end{abstract}

Colorectal cancer rates have dropped steadily for people born between 1890 and 1950, but have been increasing for every generation born since 1950. Adults born in 1990 have twice the risk of colon cancer and four times the risk of rectal cancer compared to adults born in 1950 of comparable age. A total of 16,450 new cases of colon or rectal cancers will be diagnosed this year in Americans under 50, according to the American Cancer Society. In 2014, 43 percent of colorectal cancer cases in those under 50 were in individuals

This article is freely accessible online.

Correspondence to: Dr. Steven Lehrer, Box 1236 Radiation Oncology, Mount Sinai Medical Center, 1 Gustave L. Levy Place, New York 10029, U.S.A. Tel: +1 2127657132, e-mail: steven.lehrer@ mssm.edu

Key Words: Polio virus, colorectal cancer, the UCSC Genome Browser, The Cancer Genome Atlas, cancer vaccines. aged 45 to 49 , with a rise in colorectal cancers in adults as young as their 20 s and 30 s (1).

No one knows what may be driving the rising number of cases in young people. Lifestyle, environmental or genetic factors, obesity, cigarette smoking, a diet high in red or processed meats, and lack of physical activity have been tied to increased risk.

In a previous study, we analyzed poliomyelitis incidence in US states, before the introduction of the polio vaccine. We found that polio might protect against colorectal cancer. Polio is predominantly an enteric viral infection that was progressively eradicated in the United States after the introduction of polio vaccine in the early 1950s. We noted an inverse correlation between polio incidence in US states, 19371951, and colorectal cancer incidence (2005-2009). The same inverse correlation pertained for colorectal cancer deaths (2).

Oral polio vaccine was given in the US until 2000 when a switch was made to injectable form only, which is still used. The reason for the switch was reversion of the oral vaccine to virulence and vaccine-induced polio. Very few people now in their 20 s and 30s have been vaccinated with the oral vaccine, which produces enteric inflammation comparable to wild-type polio virus and could protect against colorectal cancer. The majority of people have been vaccinated with the injectable form, which produces no intestinal inflammation at all and does not protect against colorectal cancer. Even when the oral vaccine was used, between 1960 and 2000, the coverage was $80 \%$ (3). So $20 \%$ of the population born in this period would be vulnerable to early colorectal cancer.

To further elucidate the role that poliovirus might play in colorectal cancer, the full human genome was analyzed for poliovirus sequences with the UCSC Genome Browser.

\section{Materials and Methods}

The UCSC Genome Browser is an on-line genome browser of the University of California, Santa Cruz (UCSC). The browser is an interactive website offering access to genome sequence data from a variety of vertebrate and invertebrate species and major model organisms, integrated with a large collection of aligned annotations. 


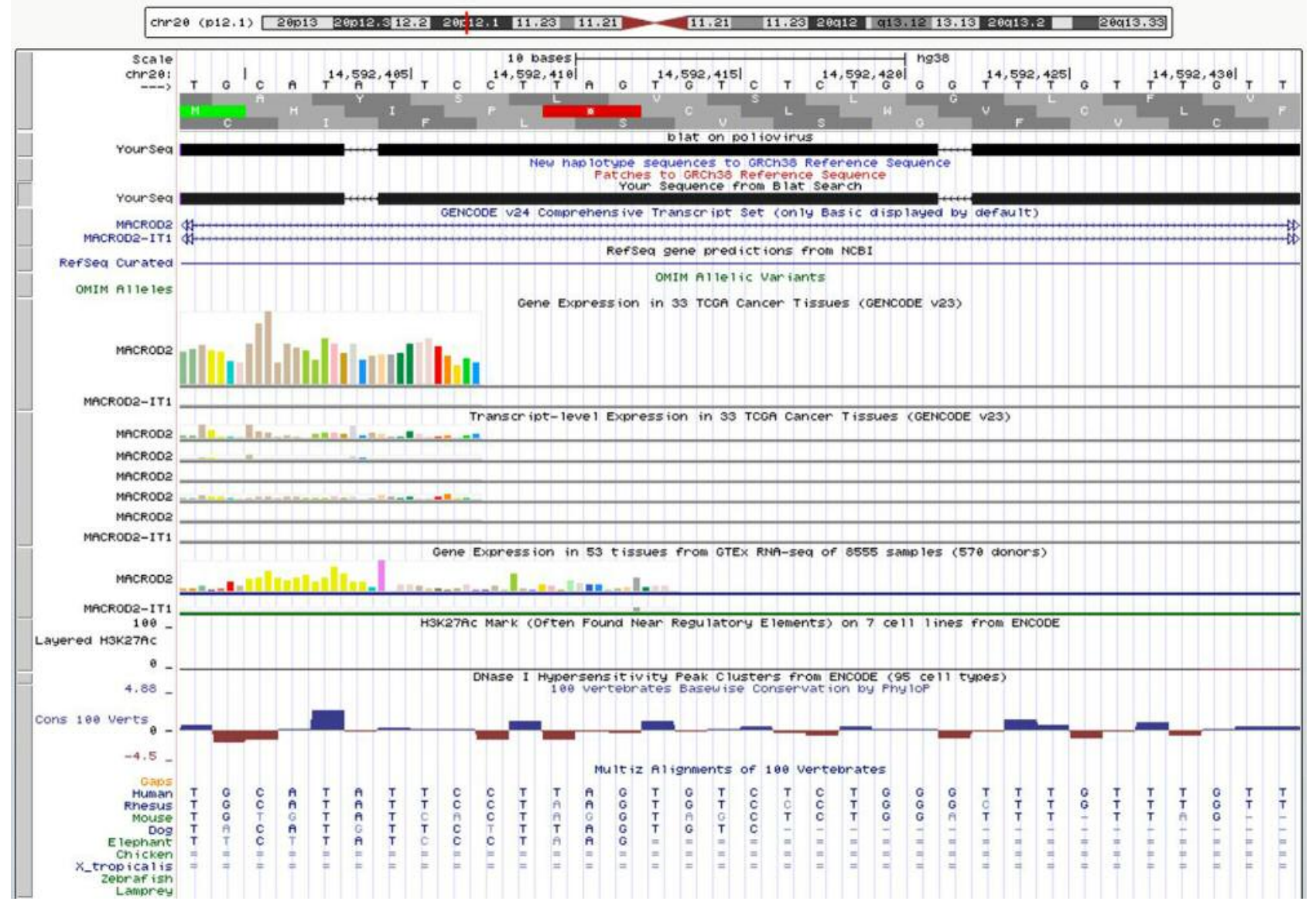

Figure 1. In chromosome 20p12.1, a poliovirus sequence with almost 100\% identity, consisting of a sense and an antisense strand of 32 nucleotides (6380 to 6411), which codes for the 3Dpol, an RNA dependent RNA polymerase whose function is to make multiple copies of the viral RNA genome. The viral sequence is in an intronic region of the MACROD2 gene, indicated by the dashed lines of Gencode. Red line: Genome and poliovirus sequence have different bases at this position. Green line: The poliovirus sequence appears to have a polyA tail that is not aligned to the genome (https://genome.ucsc.edu).

The graphical viewer is optimized to support fast interactive performance and is an open-source, web-based tool suite build on top of a MySQL database for rapid visualization, examination, and querying of data at many levels. The Genome Browser Database, browsing tools, downloadable data files, and documentation are all accessible on the UCSC Genome Bioinformatics website (https://genome.ucsc.edu) (4).

To compare the poliovirus genome to the human genome, we used BLAT, the Blast-Like Alignment Tool of the UCSC Genome Browser (4). BLAT can align a user sequence of 25 bases or more to the genome. Because some level of mismatch is tolerated, crossspecies alignments may be performed provided the species have not diverged too far from each other; this capability allowed comparison of the poliovirus genome to the human genome. The poliovirus sequence we analyzed with BLAT was FASTA Poliovirus, complete genome NCBI Reference Sequence: NC_002058.3.

Because of the immunologic basis for colorectal cancer (5), the GISTIC (Genomic Identification of Significant Targets in Cancer) module in cBioportal was used to access data in The Cancer Genome Atlas (TCGA). The GISTIC module identifies regions of the genome that are significantly amplified or deleted across a set of samples. cBioPortal for Cancer Genomics provides visualization, analysis and download of large-scale cancer genomics data sets. The Cancer Genome Atlas is a project that began in 2005 in order to catalogue genetic mutations responsible for cancer, using genome sequencing and bioinformatics.

\section{Results}

BLAT revealed three poliovirus sequences in three chromosomes:

- In Chromosome 20p12.1, a poliovirus sequence, with almost $100 \%$ identity, consisting of a sense and an antisense strand of 32 nucleotides (6380 to 6411) which codes for the 3Dpol, an RNA dependent RNA polymerase whose function is to make multiple copies of the viral RNA genome (Figure 1) (6). The viral sequence is in an intronic region of the MACROD2 gene. MACROD2 is moderately expressed in 
colon tissue (Figure 2). A similar viral sequence was present in humans and rhesus monkeys.

- In Chromosome 1p13.3, a poliovirus sequence with $89.3 \%$ identity, consisting of a sense and an antisense strand of 28 nucleotides, 811 through 838 of the virus, which codes for the VP4 protein of the viral capsid (Figure 3) (7). The sequence is within an intronic region of the solute carrier family 25 member 24 gene (SLC25A24). Increased RNA expression of the sequence was associated with significantly increased survival of colon cancer patients in TCGA (Figure 4).

- In Chromosome 2p25.1, a poliovirus sequence with almost $100 \%$ identity, consisting of two sense strands of 20 nucleotides, 7106 through 7125 of the virus, which codes for the 3Dpol. A similar viral sequence was detected in humans and rhesus monkeys. (Figure 5).

With cBioportal, the COAD cohort (adenocarcinoma of the colon) was analyzed, and the relationship of PVR, the poliovirus cellular receptor, to FCAR, a protein coding gene that encodes a receptor for the $\mathrm{Fc}$ region of Immunoglobulin A, was examined. A significant correlation in copy number alterations of both genes was found (Figure 6).

As mentioned above, we noted a significant inverse correlation between polio incidence, 1937-1951 and colorectal cancer incidence (2005-2009). The same inverse correlation pertained for colorectal cancer deaths (Figure 7) (2).

To exclude the effect of the quality of reporting by the States (good for some States, mediocre or poor for others), the multivariate general linear model (GLM) of SPSS was used (8). Based on the Annual Supplement Reported Incidence of Notifiable Diseases in the United States, 1960 (9), the relationship of scarlet fever/streptococcal sore throat to male and female colorectal cancer deaths (2005-2009) was analyzed. Any relationship of scarlet fever/streptococcal sore throat to colorectal cancer should be a chance one brought about by variations in State reporting. The relationship of polio incidence to male colorectal cancer deaths $(p<0.0005)$ and female colorectal cancer deaths $(p=0.001)$ was significant in the GLM. The relationship of scarlet fever/streptococcal sore throat to male colorectal cancer deaths $(p=0.395)$ and female colorectal cancer deaths $(p=0.316)$ was not significant.

\section{Discussion}

Eight percent of human DNA comes from human endogenous retroviruses (HERV), and some human diseases have been attributed to this DNA. HERV sequences have occasionally been adopted to serve a useful purpose, such as in the placenta, where they may safeguard fetal-maternal tolerance (10). Some HERV sequences have oncogenic properties; yet treating cancer cells with methyltransferase inhibitors increases HERV RNA and DNA. Like infectious agents, the

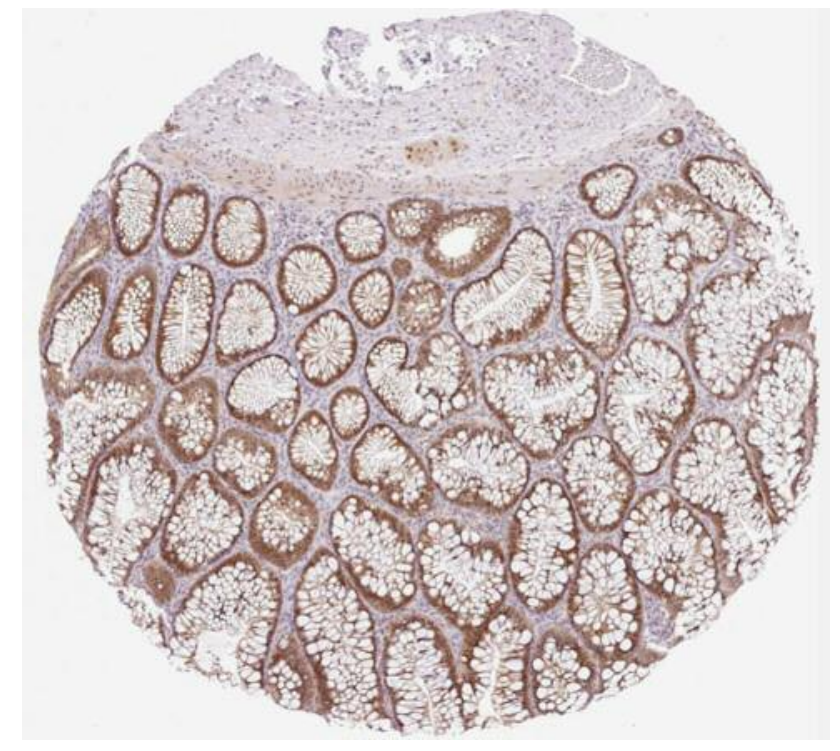

Figure 2. Colon adenoma stained with HPA049076 anti-MACROD2 antibody produced in rabbit (Human Protein Atlas available from www.proteinatlas.org).

HERV-derived nucleic acids in the cytoplasm activate innate immune responses that produce tumor cell apoptosis $(11,12)$.

Expression of the human endogenous retrovirus (HERV)-H family is associated with colorectal carcinomas. One study identified colorectal cancer specific HERV-H sequences in all stages, along with correlations between HERV-H expression, lymph node involvement, and microsatellite instability (13).

Poliovirus is a human enterovirus and member of the Picornaviridae family. Human rhinovirus type 2 (HRV2) is another member of this family. Poliovirus infects human cells by binding to an immunoglobulin-like receptor, CD155, also known as the poliovirus receptor (PVR), on the cell surface. Interaction of poliovirus and CD155 facilitates an irreversible conformational change of the viral particle necessary for viral entry. The 5' end of poliovirus RNA is extremely long - over 700 nucleotides - and highly structured. This region of the viral genome is called internal ribosome entry site (IRES), and directs translation of the viral RNA. Genetic mutations in IRES prevent viral protein synthesis (14).

The three HERV poliovirus sequences that were identified in this study in the human genome on chromosomes 20p12.1, 1p13.3 and 2p25.1 relate to colorectal cancer as follows:

- The MACRO Domain Containing 2 (MACROD2) gene on chromosome 20p12.1 has chromosome breaks in $41 \%$ of colorectal cancers (15). The gene codes for a deacetylase that removes ADP ribose from mono-ADP ribosylated proteins. The encoded protein translocates from the nucleus to the cytoplasm upon DNA damage. MACROD2 expression predicts response to 5-FU-based chemotherapy in stage III 


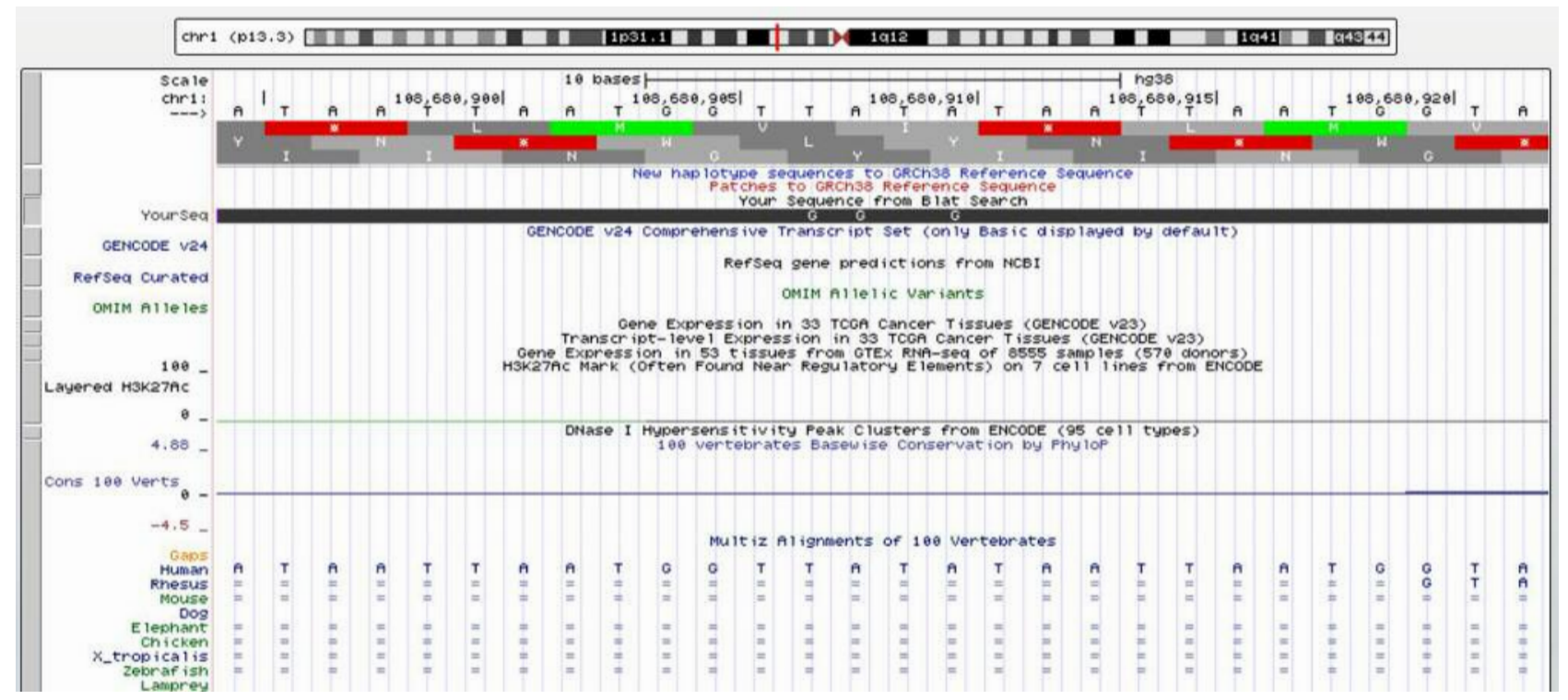

Figure 3. In chromosome 1p13.3 a poliovirus sequence with $89.3 \%$ identity, consisting of a sense and an antisense strand of 28 nucleotides, 811 through 838, which code for the VP4 protein of the viral capsid. The viral sequence is within an intronic region of the solute carrier family 25 member 24 gene (SLC25A24). Red line: Genome and poliovirus sequence have different bases at this position. Green line: The poliovirus sequence appears to have a polyA tail that is not aligned to the genome (https://genome.ucsc.edu).

colon cancer (16). The poly A tail of the poliovirus is not aligned to the genome. A 3' poly(A) tail is a common feature of picornavirus RNA genomes and the RNA genomes of many other positive-strand RNA viruses (17).

- The gene SLC25A24 on chromosome 1p13.3 is part of the SLC family. Proteins in the SLC family transport various compounds across cell membranes and component parts. The protein produced by the SLC25A24 gene carries molecules across the inner membrane of the mitochondria. The protein is an ATP-Mg/Pi carrier, transporting ATP molecules that are bound to magnesium $(\mathrm{Mg})$ through the mitochondrial inner membrane in exchange for adding or removing phosphate atoms from the mitochondria. The exchange is essential for normal energy production, the metabolism of various molecules, and protein production within cells. SLC25A24 is involved in carcinogenesis (18) and the Fontaine progeroid syndrome (19). - The lipopolysaccharide binding protein 32 (LBP-32) gene on chromosome $2 \mathrm{p} 25.1$ is up-regulated by a factor of 2.57 in colorectal cancer (20). This gene encodes a transcription factor that functions during development.

It is not surprising that poliovirus might reduce the risk of colorectal cancer. A genetically modified poliovirus, PVSRIPO, infused directly into the brain, significantly improves long-term survival in patients with recurrent glioblastoma (21). PVSRIPO is a recombinant, live, attenuated, nonpathogenic form of the oral poliovirus Sabin type 1, in which the IRES has been replaced with the IRES from human rhinovirus type 2 (HRV2). Because of the

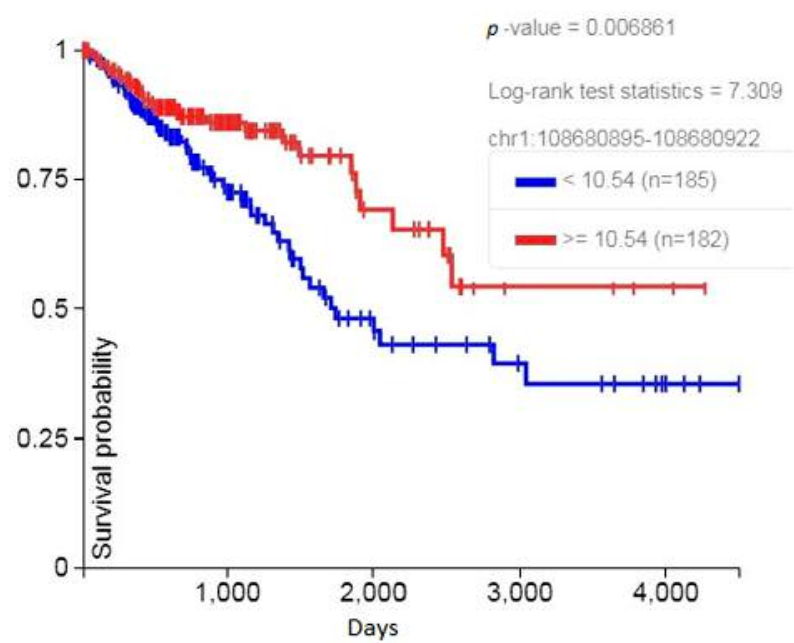

Figure 4. Increased RNA expression of the SLC25A24 gene poliovirus sequence on chromosome 1p13.3 was associated with significantly increased survival in colorectal cancer patients.

replacement, PVSRIPO cannot revert to virulence, as can Sabin type 1 virus. Most tumor cells express the PVR gene and its protein CD155, which allows tumor cells to selectively take up polio virus. The virus then replicates and produces tumor cell lysis, with an end result comparable to apoptosis (programmed cell death). 


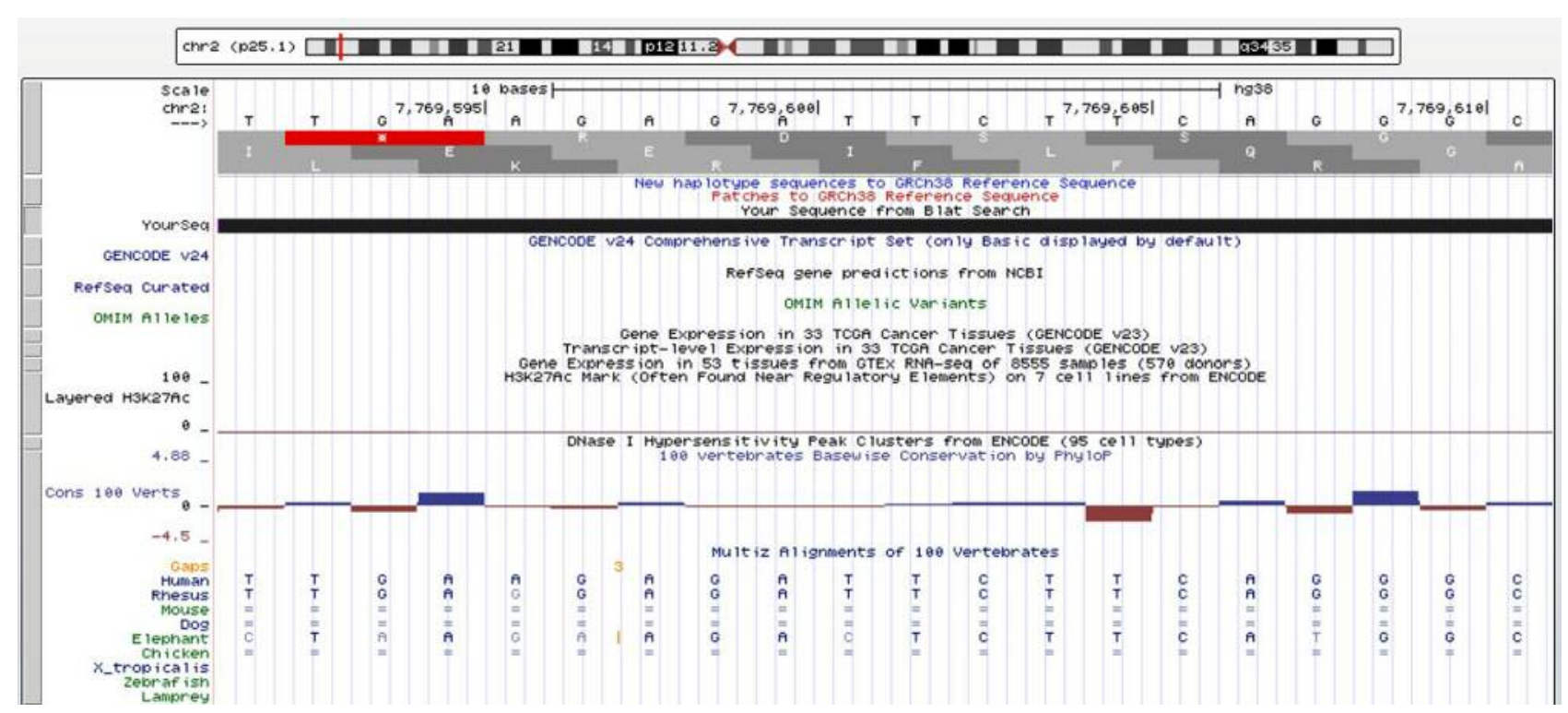

Figure 5. In chromosome 2p25.1, a poliovirus sequence with almost 100\% identity, consisting of two sense strands, 20 nucleotides, 7106 through 7125, which codes for the 3Dpol. Red line: Genome and poliovirus sequence have different bases at this position (https://genome.ucsc.edu).

Induction of apoptosis in malignant cells is a major goal of cancer therapy. Numerous apoptosis-regulating genes have been identified (22). The results of this analysis suggest that poliovirus sequences in the human genome may contribute to programmed cell death of colorectal cancer cells. In combination with an enteric poliovirus to prime the immune system, the risk of colorectal cancer may be reduced in susceptible individuals. In the case of the MACROD2 gene, enteric poliovirus might insert sequences into chromosome breaks, making colorectal tumor cells more vulnerable to apoptosis and tumor cell lysis.

A recombinant poliovirus, incapable of reverting to neurovirulence, might be given orally at intervals as a colorectal cancer vaccine to prevent colorectal cancer. Further study would be worthwhile.

\section{Conflicts of Interest}

None of the Authors have any conflicts of interest to disclose regarding this study.

\section{References}

1 Wolf AMD, Fontham ETH, Church TR, Flowers CR, Guerra CE, LaMonte SJ, Etzioni R, McKenna MT, Oeffinger KC, Shih YT, Walter LC, Andrews KS, Brawley OW, Brooks D, Fedewa SA, Manassaram-Baptiste D, Siegel RL, Wender RC and Smith RA: Colorectal cancer screening for average-risk adults: 2018 guideline update from the American Cancer Society. CA Cancer J Clin 68: 250-281, 2018.

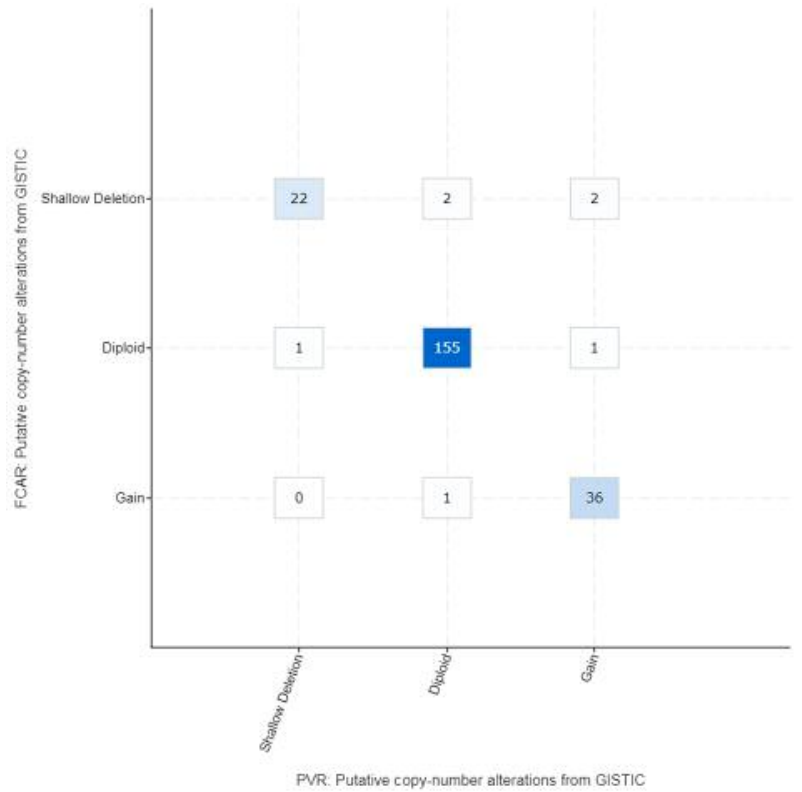

Figure 6. Analysis of the COAD (adenocarcinoma of the colon) cohort in TCGA: relationship of PVR, the poliovirus cellular receptor, to FCAR, a gene that encodes a receptor for the FC region of Immunoglobulin A. There is a significant correlation in copy number alterations of both genes. Number of cases in each box (http://www.cbioportal.org).

2 Lehrer S and Rheinstein PH: Inverse Relationship Between Polio Incidence in the US and Colorectal Cancer. In Vivo 32: 1485$1489,2018$. 

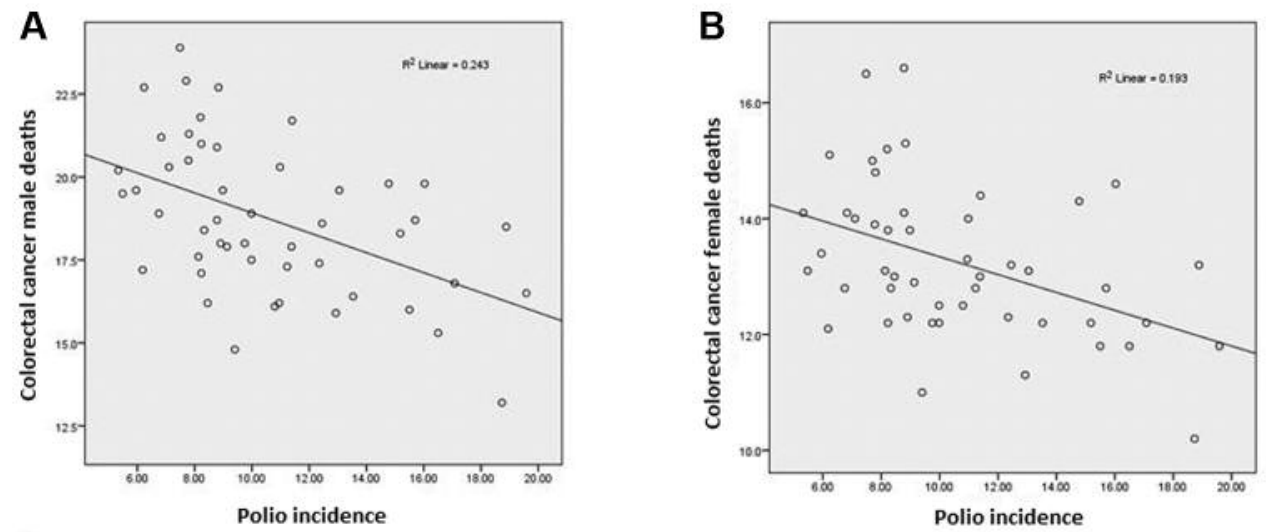

Figure 7. Polio incidence in 48 states (1937-1951) versus colorectal cancer deaths per 100,000 (2005-2009). The correlation was significant for men (A: $r=-0.493, p<0.0005)$ and women $(B: r=-0.440, p=0.002)$. Alaska and Hawaii were not States in 1951.

3 Poliomyelitis prevention in the United States: introduction of a sequential vaccination schedule of inactivated poliovirus vaccine followed by oral poliovirus vaccine. Recommendations of the Advisory Committee on Immunization Practices (ACIP). MMWR Recomm Rep 46: 1-25, 1997.

4 Kuhn RM, Haussler D and Kent WJ: The UCSC genome browser and associated tools. Brief Bioinform 14: 144-161, 2013.

5 Le DT, Hubbard-Lucey VM, Morse MA, Heery CR, Dwyer A, Marsilje TH, Brodsky AN, Chan E, Deming DA, Diaz LA, Jr., Fridman WH, Goldberg RM, Hamilton SR, Housseau F, Jaffee EM, Kang SP, Krishnamurthi SS, Lieu CH, Messersmith W, Sears CL, Segal NH, Yang A, Moss RA, Cha E, O'DonnellTormey J, Roach N, Davis AQ, McAbee K, Worrall S and Benson AB: A blueprint to advance colorectal cancer immunotherapies. Cancer Immunol Res 5: 942-949, 2017.

6 Leveque N and Semler BL: A 21st century perspective of poliovirus replication. PLoS Pathog 11: e1004825, 2015.

7 Oh HS, Pathak HB, Goodfellow IG, Arnold JJ and Cameron CE: Insight into poliovirus genome replication and encapsidation obtained from studies of 3B-3C cleavage site mutants. J Virol 83: 9370-9387, 2009.

8 Ho R: Handbook of univariate and multivariate data analysis and interpretation with SPSS. Chapman and Hall/CRC, 2006.

9 Annual Supplement Reported Incidence of Notifiable Diseases in the United States, 1960. MMWR Morb Mortal Wkly Rep 9: $1-24,1961$.

10 Kurth R and Bannert N: Beneficial and detrimental effects of human endogenous retroviruses. Int J Cancer 126: 306-314, 2010.

11 Bannert N, Hofmann H, Block A and Hohn O: HERVs New Role in Cancer: From accused perpetrators to cheerful protectors. Front Microbiol 9: 178, 2018.

12 Wildschutte JH, Williams ZH, Montesion M, Subramanian RP, Kidd JM and Coffin JM: Discovery of unfixed endogenous retrovirus insertions in diverse human populations. Proc Natl Acad Sci USA 113: E2326-E2334, 2016.

13 Perot P, Mullins CS, Naville M, Bressan C, Huhns M, Gock M, Kuhn F, Volff JN, Trillet-Lenoir V, Linnebacher M and Mallet F: Expression of young HERV-H loci in the course of colorectal carcinoma and correlation with molecular subtypes. Oncotarget 6: 40095-40111, 2015.
14 Cello J, Paul AV and Wimmer E: Chemical synthesis of poliovirus cDNA: generation of infectious virus in the absence of natural template. Science 297: 1016-1018, 2002.

15 van den Broek E, Dijkstra MJ, Krijgsman O, Sie D, Haan JC, Traets JJ, van de Wiel MA, Nagtegaal ID, Punt CJ, Carvalho B, Ylstra B, Abeln S, Meijer GA and Fijneman RJ: High prevalence and clinical relevance of genes affected by chromosomal breaks in colorectal cancer. PloS One 10: e0138141, 2015.

16 van den Broek E, den Uil SH, Coupe VMH, Delis-van Diemen PM, Bolijn AS, Bril H, Stockmann HBAC, van Grieken NCT, Meijer GA and Fijneman RJA: MACROD2 expression predicts response to 5-FU-based chemotherapy in stage III colon cancer. Oncotarget 9: 29445-29452, 2018.

17 Steil BP, Kempf BJ and Barton DJ: Poly(A) at the 3' end of positive-strand RNA and VPg-linked poly(U) at the 5' end of negative-strand RNA are reciprocal templates during replication of poliovirus RNA. J Virol 84: 2843-2858, 2010.

18 Lytovchenko O and Kunji ERS: Expression and putative role of mitochondrial transport proteins in cancer. Biochim Biophys Acta Bioenerg 1858: 641-654, 2017.

19 Rodriguez-Garcia ME, Cotrina-Vinagre FJ, Cruz-Rojo J, GarzonLorenzo L, Carnicero-Rodriguez P, Pozo JS and Martinez-Azorin F: A rare male patient with Fontaine progeroid syndrome caused by p.R217H de novo mutation in SLC25A24. Am J Med Genet A, 2018. doi: 10.1002/ajmg.a.40496. [Epub ahead of print]

20 Bianchini M, Levy E, Zucchini C, Pinski V, Macagno C, De Sanctis P, Valvassori L, Carinci P and Mordoh J: Comparative study of gene expression by cDNA microarray in human colorectal cancer tissues and normal mucosa. Int J Oncol 29: 83-94, 2006.

21 Desjardins A, Gromeier M, Herndon JE, Beaubier N, Bolognesi DP, Friedman AH, Friedman HS, McSherry F, Muscat AM, Nair S, Peters KB, Randazzo D, Sampson JH, Vlahovic G, Harrison WT, McLendon RE, Ashley D and Bigner DD: Recurrent glioblastoma treated with recombinant poliovirus. N Engl J Med 379: 150-161, 2018.

22 Baig S, Seevasant I, Mohamad J, Mukheem A, Huri HZ and Kamarul T: Potential of apoptotic pathway-targeted cancer therapeutic research: Where do we stand? Cell Death Dis 7: e2058, 2016.

Received November 1, 2018

Revised November 19, 2018 Accepted November 21, 2018 